\section{THE DIFFERENTIAL DIAGNOSIS OF SYPHILIS AND PARASYPHILIS OF}

\section{THE NERVOUS SYSTEM.}

INTRODUCTION TO A DIScUssion ON SYPHILIS AND PARASYPHILIS OF THE NerVous System at THE MEdical SOCIETY OF LONDON ON NOVEMBER 13TH, 1911.

\author{
BY
}

F. W. MOTT, M.D., F.R.S., F.R.C.P.,

DIRECTOR OF THE PATHOLOGICAL LABORATORY OF THE LONDON

COONTY ASYLOMS; PHYSICIAN TO CHARING CROSS HOSPITAL.

Fournier was the first to express the opinion that tabes dorsalis is a post-syphilitic disease, and Erb in Germany and Gowers in this country have done more than any other neurologists to support this doctrine of Fournier. Esmarch and Jessen, and later Kjelberg, were the first to call attention to the fact that general paralysis occurred especially in persons who had suffered with syphilis; but it was Fournier who really put forward the doctrine that general paralysis was, like tabes, a consequence of syphilis, and that the two diseases were so similar in their etiology that they might probably be regarded as one disease affecting different parts of the nervous system. The inoculation of a number of general paralytics with no history or signs of syphilis with the virus of a hard chancre without any signs of infection resulting led Krafft-Ebing to postulate the dictum, "general paralysis is the product of syphilization and civilization."

When I was appointed Pathologist to the London County Asylums I found no mention of syphilis as a cause of this disease and comparatively little of other mental conditions in the annual reports. This struck me forcibly, for as a neurologist I had been greatly impressed in my hospital experience with the fact that of all the causes of organic disease of the nervous system syphilis easily occupied the first place. I found that careful inquiry in cases of general paralysis revealed the fact of antecedent syphilis much more than had been suspected; still the obvious syphilitic residua of scars of gummata and other tertiary manifestations in the body were relatively infrequent. I found that cases of syphilitic brain disease with dementia were called general paralysis, also cases of Korsakoff alcoholic psychosis; but etiologically and pathologically, especially in the light of recent research these are quite different diseases and can usually be differentiated clinically. A number of causes were asserted to be the agencies capable of inducing general paralysis, and while not denying that alcoholism, sexual excesses, inherited neuropathic and psychopathic predisposition, worry, and excessive mental strain-in fact all those causes which may lead to neurasthenia-are important exciting factors, yet it is my conviction that syphilis is the essential cause.

It seemed to me that the proof would be afforded if I could collect a sufficient number of cases of juvenile general paralysis in which most of these exciting factors are absent and show that they were the subjects of congenital syphilis. From the numerous inmates in the London County Asylums I was soon able to collect a large number of cases of general paralysis occurring at puberty or early adolescence, and to demonstrate that clinically and pathologically they in no essential respect differed from cases occurring in adults the subjects of acquired syphilis.

Again, later I was able to collect 70 cases of taboparalysis and compare the same with 70 cases of tabes dorsalis, and I found among these that the parasyphilitic affection may begin with spinal symptoms or optic atrophy and terminate in general paralysis, or general paralytics may, simultaneously with or successively to the onset of the cerebral symptoms, develop tabes dorsalis; and I came to the conclusion, from a research extending over three years, that there is one tabes or wasting disease of the central nervous system from an etiological and pathological standpoint. One of the strongest arguments clinically in favour of this view is the fact that the Argyll Robertson pupil is for all practical purposes only met with in these post-syphilitic diseases of the nervous system. Researches in connexion with the examination of the blood and cerebro-spinal fluid which will be referred to later confirm this view.
According to some authorities-Bosc, Lesser, Hirschl, Nageotte, and others-general paralysis and tabes are quaternary syphilis; the former authority asserts, and with some degree of correctness, that there is a general similarity even in the lesions of general paralysis and tabes to other syphilitic lesions-namely, there is an endothelial and connective tissue hyperplasia with a tendency to fibrous and sclerous formation. But there is more than this-there is a primary neuronic decay which cannot be accounted for solely by the changes in the supporting, enclosing, and nutrifying tissues, the same as can be done in syphilitic lesions.

The view I take of the pathology of parasyphilitic affections is that in certain acquired or congenital syphilitic individuals the durability of the neurones is greatly curtailed, so that they decay and die prematurely, thereby giving rise to a series of symptoms which may be associated either with irritation of definite nerve structures -for example, lightning pains, visceral crises, mania, and epileptiform convulsions; or with neural destruction-for example, ataxy, paraesthesia, anaesthesia, paresis, and dementia. The irritative phenomena may be the sign of increased neural irritability, due to degeneration of neurones prior to their death and loss of function. I do not, however, affirm that the lymphatic and vascular changes play an insignificant part in the process of decay and death of the neurones, especially in general paralysis. Now, it may be asked, What is the evidence to show how this neural irritation and destruction is brought about?

It is a fact that the cases of syphilitic disease of the nervous system diminish in number and gravity with each succeeding year following infection, although it must be admitted there is no essential difference in the histological changes met with in early and late syphilis of the nervous system. It is, however, comparatively very rare for parasyphilitic affections to occur within four or five years of the primary infection, and the average time is at least ten years after the appearance of the primary sore. Seeing that the great bulk of persons are affected in youth or early manhood, so the curve of incidence of onset of syphilitic affections of the nervous system reaches its maximum somewhere between 25 and 30, whereas the curve of the incidence of onset of general paralysis and tabes reaches its maximum between 35 and 40 . Whereas, on the one hand, syphilis of the nervous system produces more or less sudden obtrusive symptoms indicative of gross lesions due to meningeal irritation and vascular occlusion of a random kaleidoscopic character, with remissions and exacerbations causing new and variable paralytic and irritative phenomena; on the other hand, parasyphilitic affections are usually so insidious in origin that it may be difficult to fix the date of the onset. Again, as a rule there is a continuous progress of the disease in which remissions and exacerbations are not nearly so manifest.

Whereas on the one hand syphilis of the nervous system, especially in the early stage, yields to all forms of treatment which would cause the destruction of the specific organism, on the other hand, parasyphilitic affections are seldom arrested; and, though sometimes benefited by antisyphilitic treatment, the degree of benefit is inconsiderable when compared with the effects obtained in syphilis of the nervous system. I have myself seen no disappearance of symptoms once established in tabes or general paralysis which could be associated with the treatment. Neither could we expect that mercury or arsenic in any form could restore neurones which have undergone degeneration, although, when it is the syphilitic virus which is producing symptoms due to arterial and meningeal inflammation, the administration of these remedies gives relief by destroying the specific cause, and thus arresting and clearing up the inflammatory process before neuronic destruction to any considerable degree has taken place. If the spirochaete be the cause of these neuronic degenerations in parasyphilis, how does it come to pass that it has never been demonstrated in general paralysis or tabes? whereas it has been demonstrated by many authorities in syphilitic meningitis and arteritis. It might be said that there is an intracellular granule form, but this has to be proved. So far the existence of an intracellular granule form rests only on the slender basis of analogy of the spirochaete to the tick fever spirillum, which has been shown by Leishman to have an intracellular granule 
form. If we admit this premise, then it must be assumed that the Spirochaeta pallida has passed into a resting stage, and after a lapse of years, on an average ten, it has become active and caused inflammatory changes in the membranes, vessels, and connective tissues, with secondary irritation and destruction of the neural elements. The primary meningo-encephalitic theory was the old view of the parasyphilitic affections, but most authorities now consider that the diseases termed "parasyphilitic" are due to a primary neuronic decay with secondary changes in the membranes, vascular and neuroglial structures. This view was taken by Sir David Ferrier in his Lumleian Lectures on Tabes Dorsalis. The positive Wassermann reaction, given almost in all cases of general paralysis both by the blood and cerebro-spinal fluid, would suggest that the disease was the result of active syphilis; but whereas a case of active syphilis giving the Wassermann reaction in the blood after treatment may no longer give it, this is not the case with general paralysis according to my experience. I shall therefore assume that until further evidence of a more definite nature is forthcoming to show that these late manifestations of a previous syphilitic infection are to be regarded as quaternary syphilis of a nature similar to glossitis, it will be better to accept the term "parasyphilis " or "metasyphilis," and to explain the symptoms by a premature and progressive decay of neural systems, localized in some cases to afferent systems, as in tabes, or generalized in others, as in general paralysis."*

I have not time to discuss the possibility of there being a specific spirochaete for these parasyphilitic diseases, nor the question whether parasyphilis is a result of racial immunity by widespread syphilization; nor another view, that of Neisser, that the late manifestation of syphilis in the form of tabes and general paralysis may be the result of a modification of the action of the specific organism by the widespread use of mercury. Nor will I discuss the question of anaphylaxis as an explanation, but come to the more practical side of the question of the differential diagnosis of parasyphilis and syphilis of the nervous system.

The Differential Diagnosis of Syphilis and Parasyphilis of the Nervous System from other Diseases affecting the Brain, the Spinal Cord, and the Peripheral Nerves.

The neuroses and psychoses will first occupy my attention, for in my opinion they form a most important group of cases; important because of the difficulties arising in determining whether the symptoms are purely functional or whether they are the prodromal symptoms of grave organic disease. An individual may have had syphilis, or he may think he has had syphilis; in the former case he may deny the possibility. It is in all such dubious cases that the serum diagnosis is of the greatest value: if it is positive, there is a clear indication for treatment; if the result is negative, it is often extremely useful, inasmuch as I have observed that the assurance given to the patient, that there is no active syphilis, goes a long way to improve his general condition, and, the apprehension and mental distress being removed, the patient recovers from his neurasthenic condition.

The neurasthenia occurring in the secondary or active stage of syphilis is in no way specific in its symptoms, although it may be favoured and intensified by the action of the poison, but the effect on the mind of a sensitive person in whom the moral sense has received a profound shock by the acquirement of a loathsome and what he believes to be an incurable disease, is a most important factor. He becomes morbidly apprehensive, and he suffers in consequence with sleeplessness, loss of appetite, dyspepsia and malnutrition-a combination of factors which terminate in neurasthenia. It is of importance to diagnose between the headache and other symptoms of such an induced neurasthenia and true syphilitic headache of the secondary period, or that more serious condition premonitory of the syphilitic affection of the membranes and

* I have recently had a case in which the fluid and blood taken post mortem gave a negative reaction. Clinically the case was one of rapid general paralysis of the insane. Microscopic examination showed a very marked active and widespread destruction of the cortical neurones, marked proliferation, and swelling of the capillary endothelial cells and perivascular plasma cell infiltration, general paralysis. vessels of the central nervous system. In neurasthenia there is little real pain ; there are unpleasant sensations of tightness, heaviness, emptiness, muzziness of the head, but not true pain. In syphilitic disease, on the other hand, the pain is intense, lancinating, boring, tearing-pain which is so severe as to interfere with occupation. It is often worse at night, sometimes waking the patient at a particular time. Neurasthenic headache is generally diurnal, and worse in the morning on arising and better at night on going to bed. Antisyphilitic remedies give relief in syphilitic disease, but have no influence in neurasthenia unless the condition is due in the main to a grave anaemia of the secondary period. It is very rare for a syphilitic headache to last for any length of time without serious manifestations of organic disease occurring; in fact, syphilitic brain disease seldom strikes without warning; it may be ptosis, a squint, a transitory condition of aphasia, or paralysis, a girdle sensation, etc. In neurasthenia and hysteria there are many and varied subjective symptoms. and very few, if any, objective signs of the disease.

It is important to distinguish the neurasthenic state, spinal and cerebral respectively, from tabes dorsalis in the early preataxic stage when the knee-jerks may be: present, and from general paralysis in the preparetic stage. As regards spinal neurasthenia, the knee-jerks are increased, there are no bladder troubles, there may be impotence, also attacks of giddiness, analgesia, and paraesthesia may suggest to the patient, especially if he have medical knowledge, tabes dorsalis, yet no physician would diagnose this disease in the absence of any of the characteristic pupil phenomena-namely, inequality, irregularity and rigidity or sluggish reaction to light. Seeing. the causes which lead to cerebral neurasthenia are in the subject of syphilis the very causes which predispose to paralytic dementia, it is often difficult to decide whether a neurasthenic displaying periods of depression or excitation, especially if he be the subject of chronic alcoholism, is or is not in the earliest prodromal stage of general paralysis ; for the pupil phenomena may be absent in the earliest stages of the disease, or, if not altogether absent, are certainly not nearly so definite as in tabes.

These parasyphilitic affections may be definitely differentiated from neurasthenic conditions by an examination of the cerebro-spinal fluid. The existence of lymphocytes points definitely to a chronic inflammatory condition of the central nervous system, and I have found that in practically every case of general paralysis, even in the earliest stages, a lymphocytosis occurs. Moreover, it always occurs in tabes dorsalis, therefore this fact is of great importance in the differential diagnosis. Many conditions of organic disease, however, give a lymphocyte reaction. In about half the cases of tabes dorsalis the fluid also gives a positive Wassermann reaction. In practically every case of general paralysis we have found a positive Wassermann reaction, so that a neurasthenic with slight mental symptoms whose fluid gave this reaction may be said to be suffering with general paralysis in the early stage. Comparatively speaking, only about 25 per cent. of the cases of syphilitic disease of the central nervous system give a positive reaction, though most of them give a well-marked lymphocytosis. Some nervous persons may object to have lumbar puncture. In the case of a differential diagnosis of early general paralysis from neurasthenia, an examination of the blood may suffice, for if the serum does not fix the complement and give the reaction neither will the cerebro-spinal fluid. That is to say, a negative serum reaction puts out of court general paralysis. If, however, a positive reaction of the blood is obtainable, then it is necessary, in order to decide the diagnosis, to perform lumbar puncture and examine the cerebro-spinal fluid; for it is obvious that every individual who gives a positive serum reaction is not suffering from general paralysis, as it only means antecedent syphilis. It would be interesting to know whether those individuals who gave a Wassermann reaction after a systematic course of energetic treatment are more liable to these late manifestations of the effects of the virus than those who, when treated efficiently, failed afterwards to give it. I should not be in the least surprised if this were so.

Hysteria and Hystero-Epilepsy.

Hysterical paralysis and hystero-epilepsy, according to Fournier and Lang, sometimes occur in the secondary stage. 
of syphilis, and the symptoms disappear under anti syphilitic treatment. The subjects of antecedent syphilis with a neuropathic predisposition are very liable to develop hysteria and hypochondriasis, and not very infrequently syphilophobia. A negative reaction of the blood will serve to allay their ungrounded fears and desire to be injected with 606. I have seen several such cases recently. If there is a positive reaction it denotes the necessity for treatment, and such a case as I shall describe illustrates the value of this serum reaction and the examination of the cerebro-spinal fluid.

Some years ago I treated a man with cerebral gummatous meningitis, and all the symptoms cleared up; a year later he came to me complaining of a slight pain in the left side. I told him that I hoped that it was nothing more than muscular pains, but he was to come and see me again if he were not better, as I could discover no anaesthesia or objective signs. A week later I was asked to see him by a practitioner who regarded his case as functional. He was paralysed in the lower extremities; it had come on somewhat suddenly. Knowing the history of this patient I took him into the hospital, and for the first few days he became rapidly worse, and lost complete control over the bladder and rectum, as well as complete loss of sensibility below the level of the seventh rib. Under antisyphilitic treatment he made a complete recovery. No doubt the case was one of gummatous syphilitic spinal meningitis. I am not so sure as I was once of the hope. fulness of curability of syphilitic meningitis. I have seen necropsies in many cases that had apparently recovered under treatment, but which have relapsed, and I should advise all people who have once suffered with syphilitic disease of the central nervous system to have the Wassermann reaction of the blood made periodically with a view to renewal of treatment if the reaction is positive. Not that I would say, if the patient showed any clinical signs pointing to syphilitic brain or cord disease and yet gave a negative reaction, he should not be treated, for we do not quite know all the conditions that may prevent the reaction occurring in some individuals. In illustration of this I may mention the following case.

A man of 35, who denied infection and showed no positive signs on his body, was admitted under my care to Charing Cross Hospital suffering with ophthalmoplegia interna and externa of the left eye; he was in a condition of drowsy stupor, and there was bilaterial facial paresis; his blood gave a negative reaction by three independent and very competent observers. Nevertheless, I said this is clinically syphilis. Energetic mercurial inunction treatment and iodide in large doses resulted in complete disappearance of all the symptoms in a month.

\section{Arterio-sclerosis.}

Seeing that syphilis is one of the most important factors in the production of sclerosis of the arteries, especially the cerebral arteries and general arterial sclerosis causing Bright's disease, it follows that, combined with nervous diseases, may be uraemic symptoms, headaches, pyrexia, twitching, and epileptiform seizures. If uraemic symptoms occur in a case with antecedent syphilis, the blood may give a positive reaction. Now the patient suffering with arteriosclerosis is usually over 50 years of age, and if he has an inborn neuropathic tendency may suffer with an involutional melancholia; he becomes irritable, suspicious, and even dangerous to himself and others; there may be exaggerated emotivity and a partial dementia, so that not infrequently such cases are regarded as general paralysis; however, unlike general paralysis, the patient retains his notions of personality and his autocritical faculty and he is more or less conscious of his failure of memory and mental enfeeblement. If he is the subject of various forms of coarse paralysis, the diagnosis is easy. It is extremely rare to obtain ankle clonus and Babinski's sign in general paralysis. Lumbar puncture would settle the diagnosis in a doubtful case; lymphocytosis and a positive Wassermann reaction would decide in favour of general paralysis. Arterio-sclerosis may especially involve the perforating arteries and cause lesions about the basal ganglia, giving rise to mimic disturbances, increased emotivity, accompanied by dysarthria and pseudo-bulbar symptoms; such cases, to my knowledge, have been mistaken for genaral paralysis.

Chronic Alcohoïism.

Chronic alcoholism and syphilis are so often associated that cases frequently present themselves of considerable difficulty of diagnosis. Absence of the serum reaction enables one, in the absence of definite signs of syphilitic affection of the nervous system and when all the symptoms accord with alcoholic poisoning, to exclude syphilis or parasyphilis-for example, in polyneuritic psychosis; but suppose the reaction is positive and symptoms and signs exist which may possibly indicate general paralysis or syphilitic disease of the central nervous system combined with alcoholism, and possibly masked by it. It is then that lumbar puncture and examination of the cerebrospinal fluid will be of great use, as a lymphocytosis will show that there is, in addition to the alcoholism, a syphilitic affection; if in addition there is a positive reaction, it points to general paralysis. The following is a good case in illustration of this :

A man, aged about 28, was said to be suffering from general paralysis, and I was asked to see him. I found that he had been drinking heavily for some time past; there was a great deal of mental confusion, loss of knowledge of time and place, personal illusions, and loss of memory of recent events. He had contracted syphilis five years previously, for which he had been treated. He had had a left-sided hemiplegic condition, from which he had recovered ; there was ankle clonus and Babinski's sign on this side. The pupils were equal, reacted sluggishly to light. I was told that his blood gave a negative reaction. I gave it as my opinion that it was not general paralysis, and that he would recover with mercurial treatment and compulsory total abstinence from alcohol. I saw him again nine months later; he had two large gummata on his head, headache, and tenderness on percussion over the forehead. Although he had been under the care of a medical man owing to his not complaining these had not been observed. A Wassermann gave a marked positive reaction. With mercury and large doses of iodide all the symptoms disappeared, and he is practically well. A differential diagnosis of mania a potu from mania of incipient general paralysis associated with drink can be decided by lumbar puncture at once, although sometimes, when objection is raised, the true nature of the disease must be allowed to declare itself, after the effects of the alcohol have had time to wear off, before a decision can be arrived at.

\section{Dementia Praecox.}

The age, mode of onset, and evolution of this disease are unlike any syphilitic or parasyphilitic affection due to acquired syphilis, but occasionally there is a difficulty in the diagnosis of a juvenile paralytic or an imbecile the subject of congenital syphilis. There is usually in dementia praecox a strong hereditary history of insanity. Nearly 80 per cent. commence before 25 . If there is any doubt, examination of the blood and cerebro-spinal fluid will settle the question.

Epilepsy with dementia, tuberculous and other forms of meningitis, disseminated sclerosis, cerebral tumours (nonsyphilitic), are diseases which may sometimes be mistaken for syphilitic and parasyphilitic affections. Here, again, the examination of the blood and cerebro-spinal fluid will in most cases allow of a differential diagnosis.

\section{The Differential Diagnosis of Syphilitic and} Parasyphilitic Affections.

The average time between syphilitic infection and onset of symptoms is ten years in parasyphilitic affections; very seldom is it under five years, whereas the converse is the case with syphilitic affections. In the severe forms of syphilitic disease of the central nervous system the greatest number of cases occur in the first few years after infection and diminish with each succeeding year. Whereas the syphilitic history is well defined and the scars of lesions are common in syphilitic disease, they are comparatively rare in the parasyphilitic affections. The onset of symptoms and course of syphilitic disease are usually rapid and subject to regressions and remissions; the onset of symptoms and the course of parasyphilitic affections are usually insidious and slowly progressive. except in some cases of general paralysis, especially those in which there are numerous seizures, causing, may be, transitory aphasias, monoplegias, and hemiplegias. The pupil and squint phenomena are common in syphilis, but Argyll Robertson pupil extremely rare. In parasyphilis pupil phenomena and strabismus are common 
Argyll Robertson pupil is rarely absent, especially in tabes and tabo-paralysis. Optic neuritis and post-neuritic atrophy are not uncommon in syphilis. A unilateral central scotoma, the other eye remaining healthy (an indication of a retrobulbar neuritis) affecting the papillomacular bundle, is indicative of a gummatous meningitis. In parasyphilis primary optic atrophy occurs in about 10 per cent. of the cases. Bladder disturbances are common in syphilis and parasyphilis. Severe headache, worse at night, stiffness of the neck, coarse paralysis of cranial nerves, paralysis of limb and face with clonus and plantar extensor response, are common as a result of syphilitic brain disease, but rarely if ever met with in general paralysis. Aphasias, anarthria, and dysarthria are common in brain syphilis, but not in general paralysis, in which the speech affection is characteristic. In fact, the term "general paresis" is much more correct for the parasyphilitic brain affection. The knee-jerks in a gummatous meningitis may be present one day, absent the next; in tabes they are in the great majority of cases absent throughout. The knee-jerks are exaggerated in the great majority of cases of general paralysis, but there is no ankle clonus or Babinski-in fact, these signs in a case of dementia, to my mind, indicate strongly syphilitic brain disease rather than general paralysis.

The Diagnosis of Tabo-Paralysis.

It is of very great importance to the patient and friends to be able to diagnose tabo-paralysis. Tabetic patients are apt to suffer with neurasthenia and mental depression and to resort to alcohol; moreover, to relieve the pains, the morphine habit may be acquired. Again, a potentially insane person may suffer with tabes and be the subject of delusions of persecution, putting an insane interpretation upon the lightning pains and visceral crises. Seeing that neither the Wassermann reaction of the blood and cerebro-spinal fluid nor the lymphocyte reaction will help in such cases, for both tabes and tabo-paralysis give these reactions, it may be difficult to decide whether the patient is the subject of oncoming tabo-paralysis. Still, in my experience an abundant lymphocytosis and a marked positive Wassermann reaction of the cerebro-spinal fluid points to a widespread disease, and therefore to involvement of the brain ; if, in addition, in such a case apoplectiform, migrainous, or epileptiform seizures not causing gross paralysis have occurred, and especially if the knee-jerk is noted to be absent on one side, and present on the other, tabo-paralysis is indicated. I may mention that about 10 per cent. of the cases of tabes dorsalis end in extension of the disease to the brain. The cerebral lesions in no way differ from those of general paralysis in the early stages.

The importance of a differential diagnosis of syphilis from parasyphilis of the nervous system cannot be overestimated from the point of view of prognosis and treatment; whereas the former yields to energetic treatment sometimes in a marvellous manner, the latter does not. At one time I found no distinction was made in many asylums between gross syphilitic brain disease with dementia occurring in young people, and general paralysis ; and the cases being regarded as incurable, treatment was either inadequate or not adopted. I show lantern slides of such cases.

I do not think in the light of pathology a place should be found in modern textbooks for syphilitic general paralysis, and I speak in great deference to our leading authority, Dr. Savage, who was the first authority in this country to recognize the association of syphilis with general paralysis and especially tabetic paralysis.

There are cases of syphilitic brain disease in which there is general syphilitic endarteritis without thrombosis; cases in which there may be at first no permanent coarse paralysis, only transitory seizures like those of general paralysis; the symptoms may be mainly psychical, although there may be headache, giddiness, and insomnia. The patient exhibits defective recollection of what has recently happened, mental fatigue, and incapacity for work; his actions are automatic, and disturbances of consciousness are frequent; he may become semi-conscious or semi-comatose, then recover in almost a complete manner, only to relapse again. The transitory character of the phenomena before the occurrence of occlusion of the arteries by thrombosis, resulting in permanent paralysis and dementing condi- tions, is especially suggestive of this form of syphilitic disease of the brain. The recogniton of this form of syphilis in its early stages is especially important. It is generally accompanied by a gummatous periarteritis and some meningitis more or less extensive, so that the cerebro-spinal fluid would give a lymphocyte reaction, and possibly a Wassermann; the serum would almost certainly give the reaction, and should be tested in all doubtful cases. I have seen numerous cases of multiple gummatous tumours of the brain with generalized arteritis and meningitis, and the principal symptoms have been a drowsy stupor with progressive dementia, but without coarse paralysis for some time. I have noticed that such cases generally show well-marked syphilitic residua on the skin or mucous membranes, and I should always hesitate to diagnose general paresis in such cases.

\section{Pseudo-Tabes.}

Cases occasionally occur of syphilitic cerebro-spinal meningitis which simulate the parasyphilitic affection very closely. I will narrate a typical case that came under my notice. A woman was sent into the wards under my care said to be suffering with tabes. She had lightning pains, girdle sensation, absence of knee-jerks, paraesthesia and anaesthesia of the lower extremities, and bladder troubles. The pupils were unequal, irregular in outline, and sluggish to light and accommodation. There was a history of her having had a severe headache some time previously, and squint and double vision. I found that she had had a healthy child four years prior to the onset of the cord symptoms; since then she had had several miscarriages. I found that the knee-jerk had come back, and I came to the conclusion that very probably, in view of the comparatively short time elapsing between possible infection and the onset of the severe headache, together with the variable character of the knee-jerk, this was a case of cerebrospinal gummatous meningitis. Lumbar puncture was performed-370 lymphocytes per cubic millimetre were found; the Wassermann reaction was negative, and the short interval, four years at most, between infection and onset, made me sure that the case was one of gummatous meningitis or pseudo-tabes. One month of mercurial inunction treatment and all the symptoms had disappeared and the fluid was free from lymphocytes. She still remains well, though it is now three years since I saw her first. This might have been thought to be a case of cured tabes. Hitherto I have dealt mostly with the diagnosis of cerebral affections; I must now briefly refer to the diagnosis of spinal cord diseases.

\section{The Diagnosis of Spinal Cord Diseases.}

Syphilitic affections of the membranes may be more or less localized so as to cause practically only spinal symptoms, but many cases regarded as syphilitic disease of the spinal cord, when the history of the illness and the symptoms are carefully inquired into will be found to have presented symptoms indicative of affection of the base of the brain-for example, they have suffered with headache, a little stiffness of the neck, transitory double vision, ptosis, etc. Indeed, it is probable that cases of meningitis syphilitica, owing to invasion of the membranes by the spirochaete, may occur when the roseolar rash appears, and there have been a few cases which indicate such an affection of the membranes while the primary sore is yet unhealed. A patient presenting himself with symptoms pointing to affections of the spinal cord may give a history of antecedent syphilis and yet not necessarily be suffering from syphilitic disease of the spinal cord. Still, as Dr. Buzzard long ago pointed out, it is astonishing how many cases of spinal cord affection in young men are due to comparatively recently acquired syphilis. I say comparatively recent, meaning thereby in comparison with tabes. The first thing to do, therefore, is to exclude syphilitic infection as a cause of spinal symptoms; the next thing is to show whether the symptoms have a causal relationship or are only coincidence. There is no clinical symptom or group of symptoms characteristic of syphilitic disease of the spinal cord, but there are certain symptoms which, if they occur in young adults particularly of the male sex, should lead one to suspect their specific origin. The early symptoms are spinal irritation, severe intercostal pains worse at night, cramps and contracture 
of limb, with pains radiating down them, bladder disturbances, disappearance and reappearance of the knee-jerks, the Brown-Séquard phenomenon; in fact, symptoms pointing to a local or more generalized meningitis or meningo-myelitis, but usually without pyrexia unless there be some complication.

As in cerebral syphilis so in the spinal disease the symptoms are particularly liable to remissions and regressions. One group of symptoms may improve, or even disappear, and another group appear, or symptoms may improve in one part of the body while progressing in another. These symptoms are due to a gummatous inflammation of the membranes associated generally with vascularitis involving veins and arteries. Consequently lumbar puncture and examination of the cerebro-spinal fluid reveal a marked lymphocytosis usually without a positive Wassermann reaction of the fluid, although the blood will almost certainly give it. Myelitis as a result of meningitis often follows the symptoms of meningitis, and may either be focal or diffuse; the former is the more common; the latter is indistinguishable from an acute myelitis due to other causes-for example, specific fevers, micro-organisms, and microbial toxins. The position and extent of the cord lesion may be inferred from the extent of the paralysis and loss of sensory function; the electrical reactions of the muscles will enable a judgement to be formed as to whether the anterior horns are affected. This leads me to say that cases have been described of poliomyelitis as a result of syphilis. I have had one such case under my care; the symptoms might resemble the acute spinal paralysis of the adult, especially if it were comparatively sudden in its onset, owing to the thrombosis of a vessel affected by endarteritis. Landry's paralysis might be mistaken for acute ascending myelitis of syphilitic origin, but in the latter sensation is affected, and if the patient survives there is a great tendency to the formation of bedsores, and many muscles may waste, showing loss of faradic irritability. Such symptoms are not met with in Landry's paralysis. If the signs and symptoms point to a focal transverse myelitis, we should seek to find whether it is primary or secondary.

Myelitis in the acute form may arise from pressure due either to a growth or diseased bone. The root pains which precede usually by some days or weeks the paraplegia, and the absence of signs pointing to disease of the spinal column or malignant growth help in a differential diagnosis. Lymphocytes of the cerebro-spinal fluid and a positive Wassermann reaction of the blood, even though the fluid is negative, would almost certainly indicate a syphilitic meningo-myelitis. As I have already pointed out, in most of these cases there is present or past evidence of some basic meningitis.

Disseminated sclerosis may result from multiple syphilitic foci due to obliterative arteritis; a positive Wassermann reaction of the blood, together with the age and history of the patient's illness, will suffice. A certain degree of lymphocytosis may occur in true disseminated sclerosis.

Hysteria and spinal neurasthenia in a syphilitic subject I have already referred to. The most important subject of differential diagnosis is between (1) tabes, (2) pseudotabes-a case of which I have already referred to-and (3) ataxic-toxic peripheral neuritis; and the chief points in making a diagnosis I have summarized in a tabular form, which I will throw on the screen. (See tabular synopsis at foot of page.)

Finally, it is necessary for me to refer briefly to Erb's spastic spinal paralysis; according to my experience, I am inclined to the belief of Oppenheim and Marie that these cases are really due to a syphilitic meningo-myelitis. The symptoms are a gradually developing spastic paresis of the leg, with increase of the deep reflexes; although the gait is extremely spastic, the muscular contraction is but slight; the bladder is early and almost constantly affected slight sensory disturbances are usually present. The course is chronic in most cases, and there is a tendency to improvement, and even to complete arrest. Erb regarded the anatomical basis of this disease to be a primary degeneration.

\section{The Histological Diagnosis.}

Medico-legal cases may arise where a histological diagnosis is required. There have been in the London County Asylums since the Workmen's Compensation Act a good few cases of patients dying in the asylums of general paralysis following injury. In some cases the injury has been slight and quite insufficient to cause the onset of the disease; in others it has been severe, and the question has arisen whether the patient was not already the subject of the disease when he met with the accident, or, indeed, whether it was not, owing to a sudden lapse of unconscious ness, the result of the disease, which occasioned, directly or indirectly, the accident or the injury. The naked-eye appearances of a well-marked case are characteristic, and I need not refer to them. The blood and cerebro-spinal fluid both give a positive reaction after death. For a considerable time past at Claybury the blood and cerebrospinal fluid of every case that dies has been examined by the Wassermann method and the reaction is compared with the result obtained during life; all cases in which the brains are not undoubtedly to the naked eye general paralytic brains are microscoped, and we have come to the conclusion that practically almost every case of general paralysis gives a positive lymphocyte and a positive Wassermann reaction. A man was admitted to Claybury and died ten days after admission. He was said to have been run over by a motor bus three months previously and to have sustained a fracture of the thigh. He was in an acute
I.

Tabes Dorsalis.

1. Average time between syphilitic in fection and onset of symptoms, ten years. Very rarely under four years. Usually only slight signs of syphilitic residua. Onset and course usually slow, insidious, and progressive.

2. Pupils frequently unequal and irregular. Argyll Robertson pupil rarely absent. Strabismus, transitory or permanent, frequent.

3. Primary optic atrophy in about 10 per cent. of the cases.

4. Bladder disturbances very frequent, visceral crises common.

5. Knee-jerks absent as a rule, even in the early stages.

6. Lightning pains in limbs. No stiffness in neck and spine. Girdle sensation and anaesthesia to light; touch in the thoracic region may be an early symptom.

7. Lymphocyte reaction of the cerebrospinal fluid as a rule not marked in the
early stages. Wassermann reaction in about half the cases. Blood serum always positive.
II.

Pseudo-tabes Syphilitica.

Average tima between syphilitic in fection and onset of symptoms eighteen fection and onset of symptoms eighteen months to two years, rarely after five years. Generally signs on the body of usually rapid and subject to regressions and remissions.

2. Various oculo-motor paralyses common ; Argyll Robertson pupil absent.

3. Optic neuritis and post-neuritic atrophy not uncommon. Unilateral central scotoma, due to affection of the papillo-macular bundle by the gummatous meningitis, may occur. Headache.

\section{Bladder disturbances common.}

5. Knee-jerks variable, one day absent, and then returning.

6. Spinal pains and tenderness, stiffness of the neck; the pains lancinating and radiating from the spine down the limbs. Girdle sensation and thoracic anaesthesia very frequently present.

7. Marked lymphocytosis of cerebrospinal fluid. Wassermann reaction less frequent. Blood reaction always present.
III.

Ataxic-toxic Peripheral Neuritis. 1. A history of some toxic conditions, for example, diphtheria, typhoid, lead,

2. There is pain and tenderness of the limb, and ataxic paraplegia or simply ataxia; there may be paraesthesia; the muscles are often wasted, and a reaction of degeneration may be given. Usually there is foot-drop or wrist-drop. There is no oculo-motor paralysis as a rule, and the pupils are not affected. There are no changes in the fundus. In diphtheria there is frequently paralysis of the muscle of accommodation. As a rule, there is no bladder trouble unless there is dementia. The knee-jerks may be present or absent. A serum reaction may be positive in a subject of antecedent syphilis, but the absence of cedent syphilis, but the absence of lymphocytosis of the cerebro-spinal fluid and a negative Wassermann reaction due to syphilis or parasyphilis of the spinal cord. 
maniacal condition, and he was certified as dying of acute delirious mania, which accorded with his symptoms. I found that his blood and fluid both gave a positive reaction, and there was an abundant lymphocytosis of the fluid. There were scarcely any naked-eye signs of general paralysis ; in fact, there was nothing which could be asserted positively as evidence of the disease-a circumstance which accorded well with the history, that just prior to the accident he had been discharged from the navy and had passed as a taxi driver. I found a definite scar of a chancre on the penis. Examination of several regions of the brain were made, but only in one region examined were there the histological appearances characteristic of the disease-namely, increased vascularity, well-marked infiltration of the small vessels of the cortex with plasma cells and lymphocytes, also the membranes, proliferation of the glia cells, absence of the tangential fibres, distortion of Meynert's columns, and atrophy of the constituent ganglion cells; lastly, the existence of the "rod cells of Alzheimer." All these conditions showed that this man was suffering with acute mania of general paralysis in its earliest stage; it is, therefore, highly probable that the severe injury had been the exciting factor in the production of the disease. We are able to decide two points by this examinationnamely, that the man had general paralysis, a disease that is invariably fatal ; that he had syphilis, which is an essential cause. That the evidence post mortem was to the effect that it was in its earliest stage, and might therefore never have occurred if he had not had the severe accident. The fact of the existence of the scar of the chancre and antecedent syphilis should not weigh in the minds of the jury if they were satisfied that the man in all probability was not suffering with general paralysis prior to the accident, for only a very few syphilitic subjects die of general paralysis.

Another case has recently occurred which, from a medico-legal point of view, is of interest in showing how necessary it is to examine various parts of the brain histologically to confirm the diagnosis of general paralysis. Dr. Bond, of Long Grove Asylum, recently gave evidence in a trial in which the wife of one of his patients alleged that traumatism was the cause of her husband's mental breakdown. Dr. Bond considered that the case was clinically one of general paralysis of the tabetic variety, although he said there were certain anomalous bodily symptoms. He sent me the central nervous system of this patient when he died. I found a typical tabetic lesion of the posterior columns of the spinal cord. Examination of the prefrontal and central convolutions did not show the typical lesion of general paralysis. The tip of the first temporal convolution, especially on one side, showed all the characteristic lesions which $I$ have just described. I will show photographs illustrating these points.

In conclusion, I beg to thank you for so kindly listening to me so long, and yet $I$ feel there are many things left unsaid which I might have said; however, many eminent authorities are about to follow who will be able to fill up the gaps, and very probably also from their wide experience modify and correct some of the statements I have made. Finally, I wish to acknowledge my indebtedness to my assistants, . Dr. Candler and Mr. Sydney Mann, who have carried out the researches on the blood and cerebrospinal fluid to which I have referred, and upon which I have based my opinion as to the value of the reaction. These were published in the Lancet recently by Dr. Candler.

THE Council of the Central and Associated Chambers of Agriculture at its meeting on October 3lst decided to ask the President of the Board of Agriculture to issue regulations for the sale of condensed machine-skimmed milk, and requiring that every tin should have printed upon the label "Unfit for infants and invalids."

A MeEting of the Medico-Psychological Association of Great Britain and Ireland will be held at the house of the Medical Society of London (Chandos Street), under the presidency of Dr. W. R. Dawson, on Tuesday next, at 3 p.m. After the transaction of formal business, papers will be read by Dr. Geoffrey Clarke on sterilization from the eugenic standpoint, and by Dr. C. T. Ewart on amenorrhoeal insanity.

\section{CHOLERA AT PALERMO :}

Further Experience in the Treatment of Cholera

BY InJections of Hypertonic Salines aNd

Permanganates Internally among Europeans at Palermo.*

By LEONARD ROGERS, M.D., MaJor I.M.S.

IT is impossible, as it is unnecessary, in such a paper as this, to give a complete account of all the details of the system of treating cholera which I have worked out during the last few years in Calcutta, as they are fully recorded in my recently published work on the disease, ${ }^{1}$ while a summary appeared in a paper in the BrITISH Medical Journal.

Pathological Principles.

It will, however, be advisable briefly to recapitulate the principles on which it is based, so as to allow of what I have to say regarding my recent very instructive experience of cholera among Europeans at Palermo being more readily followed.

The Blood Changes in Cholera as a Scientific Basis for its Treatment.

In the first place my researches ${ }^{3}$ have shown that in the collapse stage of cholera, from one-half to two-thirds, or even more, of the fluid of the serum has been lost to the system, while in the most severe cases a still greater proportion of the chlorides may be lost, and the serum may even be haemolyzed. These observations indicate the necessity of rapidly replacing the lost salts as well as the lost fluid, and of raising the salts in the blood somewhat above the normal point, so that the osmotic currents will tend to cause fluid to run into the blood rather than out of it, and thus the diarrhoea will be checked, instead of increased, as it is by the use of normal salines. For this purpose I use the following hypertonic saline solution :

\section{Sodium chloride \\ Potassium chloride \\ Calcium chloride ...}

Aqua

120 grains.

As this formula contains 1.35 per cent. of sodium chloride it is twice as strong as formerly used as normal saline, and half as strong again as the amount of salts now given as the normal amount by physiologists. The last two chlorides are added to make the proportion of the three approximate to Ringer's solution, which, when perfused through an animal's heart, will keep it beating very much longer than sodium chloride alone. Messrs. Parke Davis make hypertonic tablets of the above composition such that four to one pint make my hypertonic solution, while three in the same amount make an approximately normal saline. I used them with marked success at Palermo. I have, however, used 2 drachms to the pint of sodium chloride alone in very many cases, with successful results. I may take this opportunity of pointing out that the solution advised for intravenous injection in the article on cholera in the last edition of Allbutt's System of Medicine, consisting of 0.4 per cent. sodium chloride and 0.2 per cent. sodium carbonate, is dangerously hypotonic; in fact, I found it haemolyzed a little of my own blood nearly completely in about two hours, so that its use in large quantities would probably have most disastrous consequences.

\section{Blood Pressure and Specific Gravity Estimations as a} Guide to Transfusions in Cholera.

The degree of collapse may be accurately measured by taking the blood pressure with one of the numerous modifications of the Riva-Rocci mercury manometer. Observations on several hundred cases of cholera have shown that if the blood pressure has fallen to $70 \mathrm{~mm}$. or less in natives of India, or to $80 \mathrm{~mm}$. or less in Europeans-which means a serious degree of collapse-it is necessary to give intravenous injections, but if above these points, saline injections are readily absorbed when given subcutaneously as hypertonic solutions, or by the rectum as isotonic ones. of even greater value is the estimation of the specific gravity of the blood by means of a series

\footnotetext{
* Read before the Clinical Section of the Royal Society of Medicine.
} 